\title{
Model study of a surfactant on the GaAs (100) surface
}

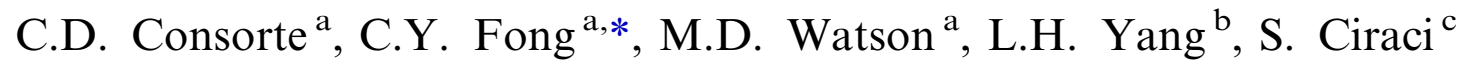 \\ a Department of Physics, University of California, Davis, CA 95616-8677, USA \\ ${ }^{\mathrm{b}}$ H-Division, Lawrence Livermore National Laboratory, Livermore, CA 94551, USA \\ ${ }^{\mathrm{c}}$ Department of Physics, Bilkent University, Bilkent, Turkey
}

\begin{abstract}
Based on the facts that: (a) the transverse acoustic vibrational branch frequency is softened at the Brillouin zone boundaries of crystalline GaAs; (b) at the surface, the Ga-As bond is stronger than Ga-Te bond; and (c) the requirement that the final bond orientation of the Te surfactant should be rotated by $90^{\circ}$ with respect to its initial orientation, we carried out a model study of an exchange process in epitaxial growth of GaAs (100). Even with very restrictive conditions imposed on the atomic movements, this study explains why Te is an effective surfactant for this type of growth.
\end{abstract}

(C) 2002 Elsevier Science B.V. All rights reserved.

Keywords: GaAs (100) surface; Atoms; Monolayers

\section{Introduction}

In 1989, Copel et al. [1] demonstrated that significant improvement in sample quality could be achieved for epitaxial growth of germanium $(\mathrm{Ge})$ on the silicon $(\mathrm{Si})$ (100) surface by a priori adsorbing arsenic (As) or antimony ( $\mathrm{Sb}$ ). These atoms segregate to the front of the growth plane and serve as surfactants. Thus, a new way to enhance two-dimensional (2D) epitaxial growth was introduced. Since then, the surfactant mediated epitaxial (SME) growth method has attracted much attention and has been extended to III-V compounds and related heterojunctions [2-5]. Among these extensions, Grandjean and Massies (GM) provided evidence that, for epitaxial growth of GaAs along the [100] direction, tellurium (Te) is an effective [3] surfactant. GM proposed an exchange [4] between the surfactants and group $\mathrm{V}$ atoms but did not give any detail. The initial conditions for their experiments are: (1) Te atoms occupy substitutional As sites at the surface; (2) the coverage of $\mathrm{Te}$ atoms is about $0.5-0.6$ monolayers (ML); and (3) both As and Ga adatoms are present on top of the Te atoms with high As coverage $(\sim 0.7 \mathrm{ML})$.

\footnotetext{
* Corresponding author

E-mail address: fong@physics.ucdavis.edu (C.Y. Fong).
}

They determined $\Delta E$, the energy barrier of the exchange process to be $15 \mathrm{meV}$ ( 1.0 mRy).

Several calculations have subsequently been made concerning $\mathrm{Te}$ acting as a surfactant during GaAs related growth. Miwa and Ferraz [6] studied the dimer exchange process involved in the growth of a GaAs $\mid$ InAs interface along the [100] direction. Gundel and Faschinger [7] (GF) determined possible Te sites on GaAs (100). Both of these calculations used the local density approximation [8] of density functional theory [8]. Pseudopotentials [9] were used for the ionic potentials and plane waves were used as the basis functions. The number of plane waves was determined by a cutoff energy. Miwa et al. used $12 \mathrm{Ry}$ and GF used $15 \mathrm{Ry}$. GF showed that a Te atom is bound to two Ga atoms near the top of the surface, in qualitative agreement with the experiment [3]. Miwa et al. suggested that the exchange process is between an As and a Te dimer, and is a second neighbor process that can occur only after deposition of both In and As atoms. The presence of In adatoms is consistent with the aforementioned initial condition (3). However, the surfactant atoms were ultimately located directly above surface atoms, and their results were based upon a comparison of the total energy before and after this direct exchange. Because both GaAs and InAs form the zinc-blende structure, a direct exchange between surfactant (Te) dimers and addimers (As) will not allow sustained epitaxial growth. Thus, neither a 
microscopic description of the origin of the exchange process nor a mechanism by which the process continues has been determined.

In this paper, we provide a microscopic description of a possible exchange process between Te surfactant atoms and incoming As adatoms on the GaAs (100) surface that is energetically allowed and capable of perpetuating layer-by-layer growth. We used a cluster model approach, because: (1) there still is a controversy about the reconstruction of the GaAs (100) surface when exposed to Te atoms [3,7]; and (2) we intend to explore, without contradicting the experimental conditions, the smallest region on the surface on which the exchange process can occur based on the experimental fact that the exchange process is very efficient [3]. All of our calculations were performed using the same method as in Ref. [6] except that we determined that an energy cutoff of $25 \mathrm{Ry}$ was necessary to reduce the uncertainty in the total energy to less than $1.0 \mathrm{mRy}$.

Our 24-atom reference cluster (shown in Fig. 1a) is designed to simulate a section of the surface, including a Te atom. We arranged $4 \mathrm{Ga}$ and $5 \mathrm{As}$ atoms in the appropriate tetrahedral bonding configuration (the orientation is shown in Fig. 1(a)) and saturated the dangling bonds with hydrogen $(\mathrm{H})$ atoms $(\mathrm{H}$ was given a charge of $0.75 \mathrm{e}$ when saturating As and $1.25 \mathrm{e}$ when saturating Ga). These 23 atoms, except the Te atom, form the so-called substrate. The Te surfactant atom was placed so that it substituted a surface As atom. The $\mathrm{Ga}-\mathrm{As}$ bond length was set to, and held fixed at, 4.5826 a.u. (a.u. is the bohr radius, 1 a.u. $=0.5289 \AA$ ). This value was determined from calculations of bulk GaAs and is $\sim 1 \%$ smaller than the experimental value of 4.6265 a.u. [10]. The positions of the Te and $\mathrm{H}$ atoms were then relaxed until the Hellmann-Feynman forces were reduced to less than $2.5 \mathrm{mRy} / \mathrm{a}$.u. The cluster was set in the center of a large supercell of dimensions $30 \times$ $30 \times 40$ (a.u.) [3].

Our next step was the addition of a $\mathrm{Ga}$ atom and an As atom as required by the experiment. We called them adatoms. While determining their initial configuration, we realized that when the Te atom (which is bound to two substrate $\mathrm{Ga}$ atoms) moves along the direction perpendicular to the plane defined by these three atoms (Fig. 1a), it will experience a smaller restoring force than if it were to move in the plane. This is because motion normal to the plane does not strongly distort the $\mathrm{Te}-\mathrm{Ga}$ bond length or angle subtended at the Te atom. Therefore, this shear-type of motion should cost less energy. In fact, such motion is manifested in the softening of the transverse acoustic branch frequency at the Brillouin zone boundaries of crystalline GaAs [11]. Thus, we placed the As adatom so that it can help the Te atom initiate this type of motion at the onset of the exchange. The Ga adatom was placed where the next Ga layer would form in hopes that it will help direct the Te atom
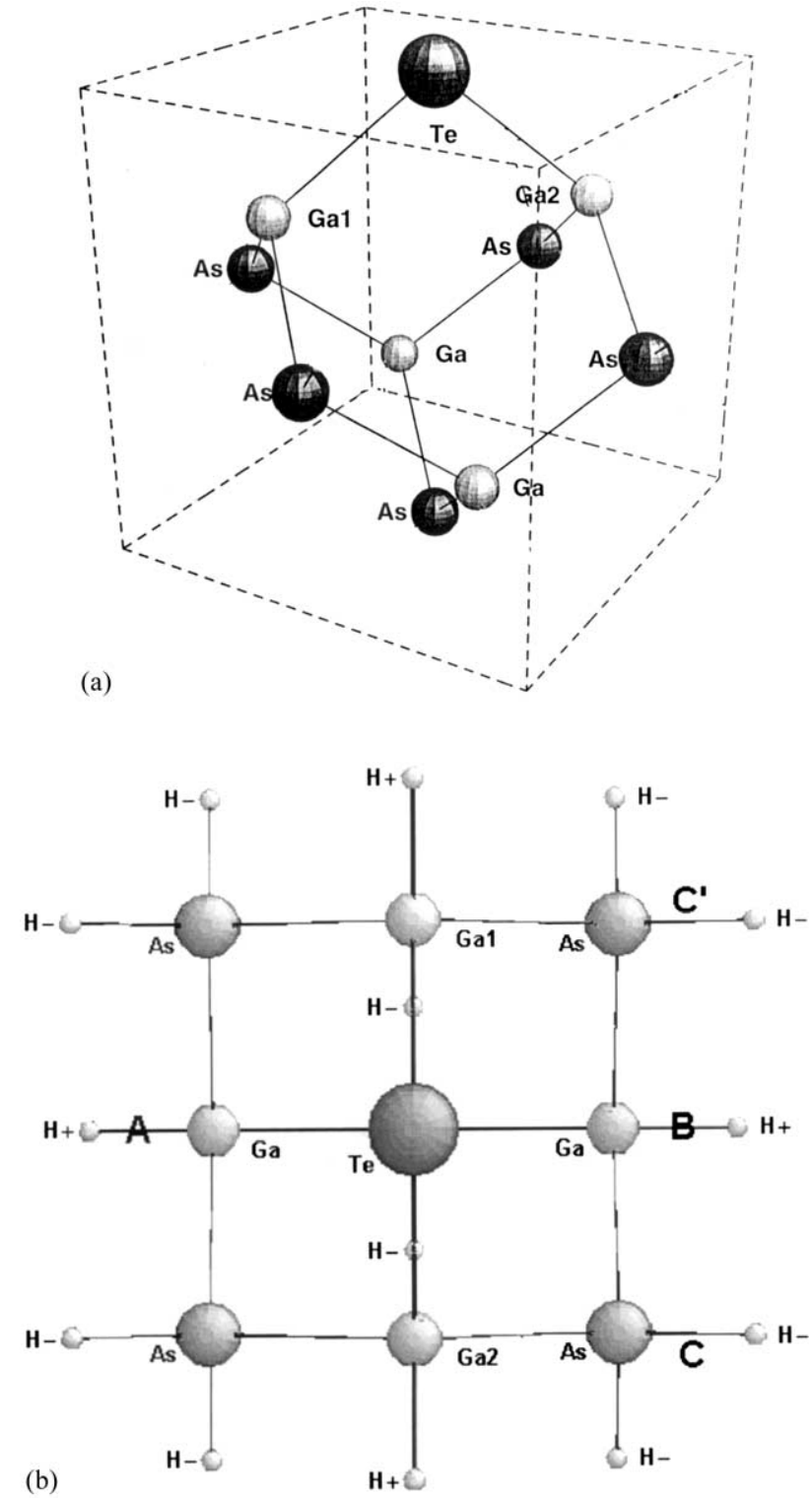

Fig. 1. (a) Cluster model in three-dimensional view. The large gray sphere is Te, the black spheres are As, the white spheres are Ga and the small gray spheres are the $\mathrm{H}$ atoms used to saturate the dangling bonds. (b) Same model viewed from [001] direction where the A and B sites are the respective initial locations of the As and $\mathrm{Ga}$ adatoms and $\mathrm{C}$ and by symmetry, $\mathrm{C}^{\prime}$ are the final locations of the Te surfactant adatom.

toward the next substitutional site. In Fig. 1b, we show the cluster from above and label the initial positions of the As (site A) and Ga (site B) adatoms. The locations of $\mathrm{A}$ and $\mathrm{B}$ are directly above their respective nearby $\mathrm{Ga}$ atoms and both are metastable positions at the surface as determined theoretically by Kley et al. [12]. Since site A is not a proper position for any As atom at the surface, there must be a reasonably large coverage of As to ensure its occupancy (experimental condition (3)). The As adatom is placed 2.773 a.u. above the level of 
Ga1 and Ga2 (Fig. 1). The qualitative features of the exchange path (described below) were not sensitive to this height as long as the initial placement of the As adatom was between the heights of the Gal atom and the Te atom.

We now define a possible exchange path that will allow the As adatom to supplant the Te atom in the surface while the $\mathrm{Ga}$ adatom helps the Te atom move to the next substitutional site, where the layer-by-layer growth process can continue. We show the final position of the Te atom as site $\mathrm{C}_{\text {or }} \mathrm{C}^{\prime}$ (Fig. 1b, directly above the nearby As atoms). Thus, there cannot be a full monolayer coverage of $\mathrm{Te}$ in accordance with experiment (condition (2)). The path was determined by first calculating the total energy of the initial configuration, then displacing both the surfactant atom and the adatoms and recalculating the total energy. If the difference in the total energy between consecutive configurations was either less than or equal to $\Delta E$, we accepted the move as a part of the path. Since this is a model approach and does not treat the temperature effects (such as small variations in the $\mathrm{Te}-\mathrm{Ga}$ adatom bond length) which can facilitate the exchange process, we imposed strict conditions on the movements of the three atoms - the Te atom and the As and Ga adatoms. In effect, we adopted the worst-case scenario. The 23 atoms simulating the substrate were held fixed in the reference configuration (Fig. 1). The three active atoms were allowed only to move expeditiously toward sites suitable for the perpetuation of layer-by-layer growth. The movements of the active atoms are shown in the snapshot series in Fig. 2.

We initiated the exchange by moving the Te atom and As adatom along the $y$-axis (Fig. 2). As we suggested earlier, this motion is perpendicular to the plane defined by the Te, Ga1 and $\mathrm{Ga} 2$ atoms. As expected, these initial movements can occur without causing any increase in energy. However, by the time the Te atom had moved 0.06 a.u. and the As adatom had moved 0.05 a.u., the total energy had increased by $1 \mathrm{mRy}$, the experimentally determined value of the energy barrier $\Delta E$, because of the interaction between the Te atom and the Ga adatom. However, the As adatom was still too far away to form bonds with $\mathrm{Ga} 1$ and $\mathrm{Ga} 2$. Since there was no other atom above the Ga adatom, we allowed it a vertical degree of freedom. Resuming the path at the point where the energy barrier was first encountered, we fixed the corresponding distance between the Te atom and the $\mathrm{Ga}$ adatom (at 4.46 a.u.) and continued the motion of the adatoms. When the Te atom and As adatom had moved 0.2 and 0.15 a.u., respectively, along the $y$-axis (step 2 in Fig. 2), the total energy was lowered by 0.038 $\mathrm{Ry}$ with respect to step 1 . Since we want the exchange process to be self-sustaining, the Te atom should move upward toward a site in the new layer while simultaneously moving to one side of the Ga adatom (along the $x$-axis in Fig. 2), so that it can reach its final position of $\mathrm{C}$ or $\mathrm{C}^{\prime}$. Between steps 2 and 4 , the As adatom continues to move directly toward the initial position of the $\mathrm{Te}$ atom and the total energy is lowered each step by $\sim 0.15$ $\mathrm{Ry}$. When the $x$-component of the Te atom reached $98 \%$ of its final value ( $x$-component of the site C) (step 5 in Fig. 2), we began to lower the Ga adatom toward the substrate. The critical step was to determine whether it was possible to equalize the heights of the Te atom and $\mathrm{Ga}$ adatom without experiencing a barrier larger than $\Delta E$. Our calculations revealed two acceptable pathways: one pathway required overcoming another $\Delta E$ energy barrier, while in the other the energy remained constant. Step 5 of Fig. 2 depicts the latter path with the height of the $\mathrm{Ga}$ adatom reduced by 0.3 a.u. relative to step 4 . The $\mathrm{Ga}$ adatom can now be easily lowered, resulting in a monotonic reduction of the total energy. The As adatom arrives at its final position with one of its two dangling bonds forming a new bond with the lowered Ga adatom. The energy difference between steps 1 and 8 is about 0.4 Ry.

Throughout the entire exchange process, a $\Delta E$ barrier appears only near the beginning and possibly in the $\mathrm{Ga}$ adatom's pathway as described above. We also found that this exchange process, and in particular the lowering of the $\mathrm{Ga}$ adatom, can proceed extremely quickly

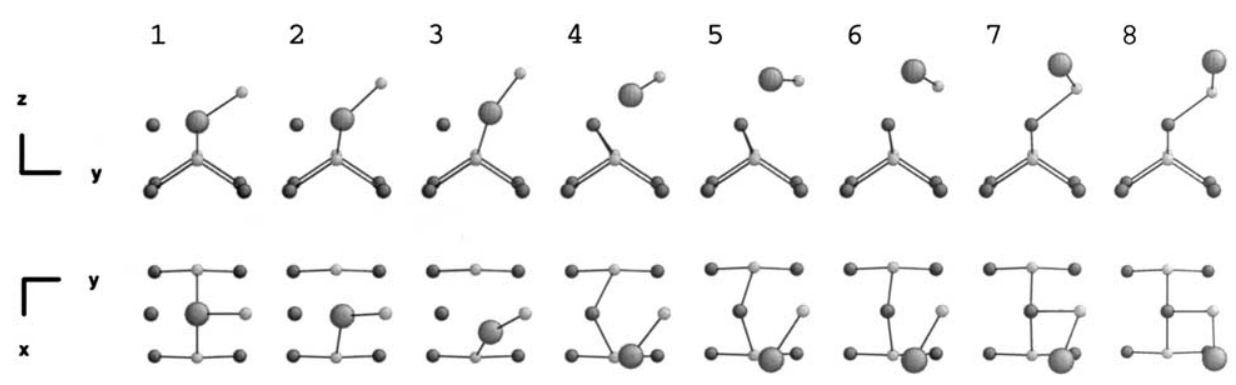

Fig. 2. A snapshot series of a pathway for the exchange process between the surfactant, Te, and the As adatom with the presence of a Ga adatom. (a) Side view; (b) top view. Large sphere is the Te, dark spheres are As and white spheres are Ga. For clarity, most of the substrate atoms have been omitted. 
if fluctuations in the $\mathrm{Te}-\mathrm{Ga}$ adatom bond length associated with finite temperature are included. This is in qualitative agreement with the experiment [3]. We should emphasize that if the $\mathrm{Ga}$ adatom were not present, the Te atom could gain enough energy to diffuse away. The weak Ga (adatom)-Te bond stops this uncontrolled diffusion process. This explains why the experiment needs the presence of $\mathrm{Ga}$ adatoms.

In summary, we have used cluster models and firstprinciples calculations to find a self-perpetuating microscopic pathway for the exchange process between $\mathrm{Te}$ surfactant atoms and host adatoms on the GaAs (100) surface. Our model is consistent with the experimental facts: (1) the Te atom initially occupies a substitutional As site at the surface; (2) the coverage of $\mathrm{Te}$ at the surface is less than a complete monolayer; (3) it is necessary to have the presence of both $\mathrm{Ga}$ and As adatoms with a larger coverage (but still less than a ML) for the As adatoms. To elaborate on (3), we found that the presence of valence III adatoms reduces the diffusion length of the surfactant atoms and facilitates growth of the next layer. Even with our strictly imposed limitations on atom movements, the path of the exchange process does not show any barrier higher than $1.0 \mathrm{mRy}$ in agreement with experimental results. This further supports the experimental claim that the exchange process can proceed very efficiently [3].

\section{Acknowledgements}

This work is supported in part by the National Science Foundation under grant INT-9872053 and TUBITAK TBAG-1668, and the Campus Laboratory Collaboration Grant of the University of California. LHY is also supported by DOE.

\section{References}

[1] M. Copel, M.C. Reuter, E. Kaxiras, R.M. Tromp, Phys. Rev. Lett. 63 (1989) 632.

[2] N. Grandjean, J. Massies, V.H. Etgens, Phys. Rev. Lett. 69 (1992) 796.

[3] N. Grandjean, J. Massies, Phys. Rev. B53 (1996) R13231.

[4] N. Grandjean, J. Massies, in: B. Gil, R.-L. Aulombard (Eds.), Semiconductor Heteroepitaxy: Growth, Characterization and Device Applications, World Science, Singapore, 1995, p. 548.

[5] J. Massies, N. Grandjean, Phys. Rev. B48 (1993) 8502.

[6] R.H. Miwa, A.C. Ferraz, Appl. Surf. Sci. 123-124 (1998) 449.

[7] S. Gundel, W. Faschinger, Phys. Rev. B59 (1999) 5602.

[8] W. Kohn, L.J. Sham, Phys. Rev. 140 (1965) A1133; P. Hohenberg, W. Kohn, Phys. Rev. 136 (1964) B864.

[9] M.L. Cohen, Phys. Today 32 (1979) 40.

[10] R.W.G. Wyckoff, Crystal Structure, vol. 1, Interscience Pub, Krieger Publishing Company, Melbourne, Florida 1986 (Reprint), 1963 , p. 110.

[11] B.N. Brookhouse, in: T.A. Bak (Ed.), Phonons and Phonon Interaction, Benjamin Inc, New York, 1964, p. 221.

[12] A. Kley, P. Ruggerone, M. Scheffler, Phys. Rev. Lett. 79 (1997) 5278. 